\title{
Attenuation correction in cardiac PET: To raise awareness for a problem which is as old as PET/CT
}

\author{
Stephan G. Nekolla, PhD, FESC, ${ }^{\text {a,b }}$ and Axel Martinez-Möller, PhD ${ }^{c}$ \\ ${ }^{a}$ Nuklearmedizinische Klinik und Poliklinik, Klinikum rechts der Isar, Technische Universität \\ München, Munich, Germany \\ ${ }^{b}$ DZKH (Deutsches Zentrum für Herz-Kreislauf-Forschung e.V.), partner site Munich Heart \\ Alliance, Munich, Germany \\ c European Patent Office, Munich, Germany
}

Received Jan 29, 2015; accepted Jan 29, 2015

doi: $10.1007 / \mathrm{s} 12350-015-0083-x$

\section{See related article, pp. 1285-1295}

Attenuation correction is a cornerstone in quantification of PET data. It ensures that some more or less arbitrary counts per seconds are converted to true Becquerel per milliliter which in turn enables the estimation of biologically relevant parameters. Basically, it allows the transformation of physical into physiological parameters. But it also ensures that any structures with a homogeneous tracer distribution appear precisely as such-homogeneous. This holds true for all PET imaging whether it is used for oncology, neurology, or cardiology. In oncological imaging, the co-reading of attenuation (AC) and non-attenuation-corrected (NAC) data is a recommended procedure. Misalignment between the emission and transmission data results in artifacts stemming from these data inconsistencies which the reconstruction algorithm typically is naturally not aware of. Fortunately, oncological imaging is focused on the detection of focal lesions and rather irregular structures. Thus, in NAC oncological images, the lesions are usually still visually detectable. Unfortunately, the same is not applicable to cardiac PET imaging. As Figure 1 shows, PET NAC data of the heart are basically useless. Without correcting for attenuation

Reprint requests: Stephan G. Nekolla, PhD, FESC, Nuklearmedizinische Klinik und Poliklinik, Klinikum rechts der Isar, Technische Universität München, Ismaninger Str. 22, 81675 Munich, Germany; stephan.nekolla@tum.de

J Nucl Cardiol 2015;22:1296-9.

$1071-3581 / \$ 34.00$

Copyright (c) 2015 American Society of Nuclear Cardiology. (and also scatter), the image intensity rapidly drops toward the image (i.e., body) center as not only a single photon (as this is the case for SPECT) but both photons from the positrons' annihilation event must escape undisturbed from physical interactions from the body. Consequentially, in cardiac imaging, a homogenous uptake (such as a normally perfused left ventricle with a relatively constant wall thickness) is not constant at all but a function of the distance to the body surface with tracer uptake in the inferior wall so massively reduced that the clinical information is almost negligible (Figure 1). As mentioned, cardiac SPECT imaging does suffer from this problem to a much lesser degree and the majority of myocardial perfusion imaging (MPI) is performed in this way. Although there is still work performed in this arena, some members of the latest generation of dedicated, fast SPECT cameras operate in non-attenuation correction mode only. ${ }^{1}$

The problem per se gained relatively rapid attention with the introduction of PET/CT systems. ${ }^{2,3}$ The fact that the CT scan took only a few seconds but the cardiac PET scan was acquired for 10-20 min introduced a systematic difference between data which were acquired over many respiratory cycles (PET) using rotating 68-Ge rod sources and one which basically represented a snapshot of a given respiratory status (CT). This CT scan was then converted to a so-called $\mu$ map to address the difference in photon absorption between the relatively low-energy $\mathrm{x}$-ray photons and the $511 \mathrm{keV}$ photon from the positron annihilation. Unfortunately, the initial idea of acquiring CT data which were also blurred over many respiratory cycles resulted in a significant radiation exposure. ${ }^{4}$ However, this study revealed that a very rapid, ultra-low-dose scan showed a similar performance to the acquisitions in a conventional 


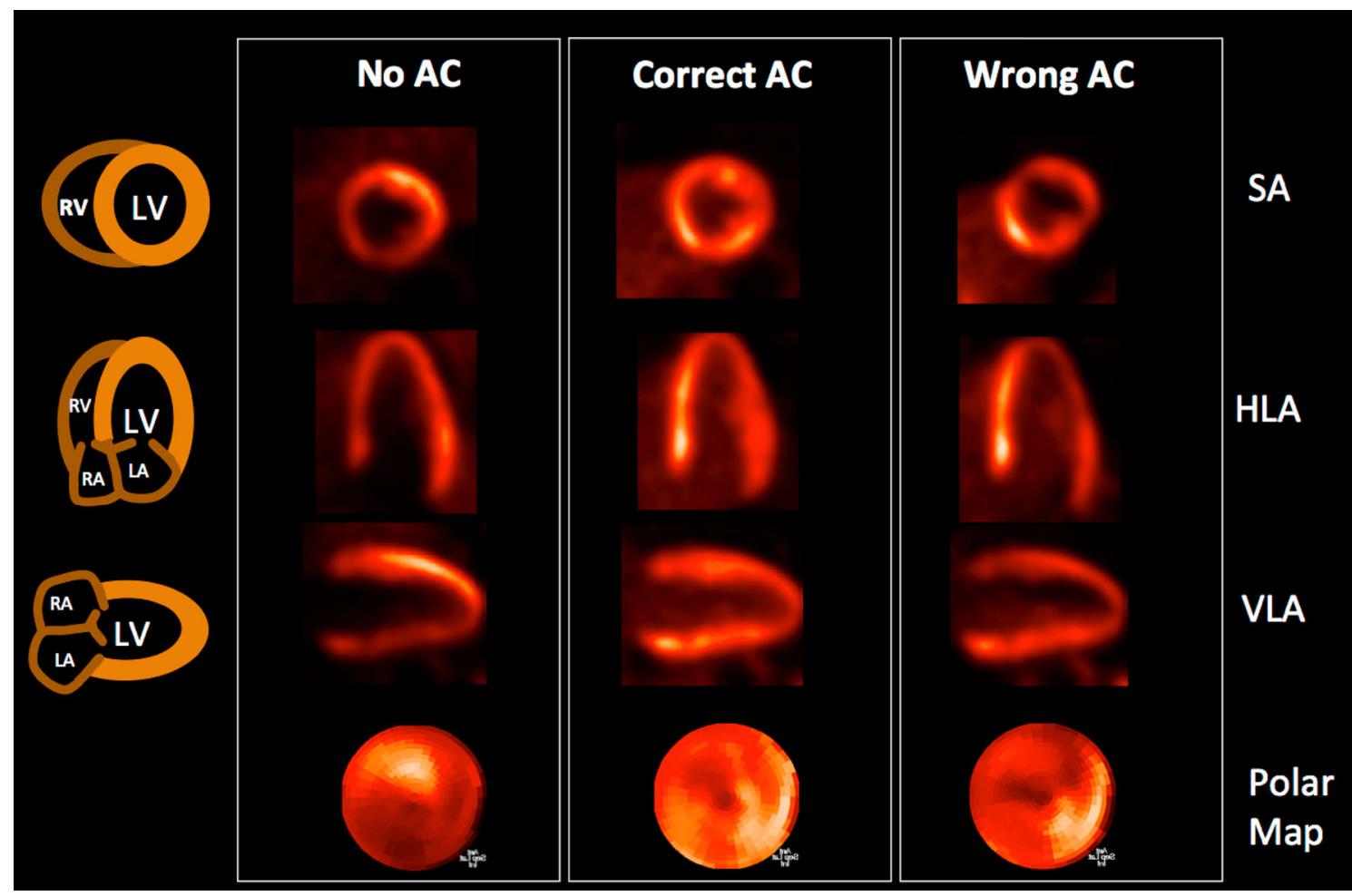

Figure 1. Example of a cardiac FDG PET/CT scan with no attenuation correction (AC), correct $\mathrm{AC}$, and using a CT misaligned by approximately $10 \mathrm{~mm}$. Whereas the non-AC scan is more or less useless, the misalignment introduces severe deviations from the correct reconstruction.

PET with rotating rod sources. Yet, it was very fast clear that a correct alignment was mandatory as the PET/CT cardiac data as acquired resulted in clinically relevant artifacts in $20 \%-30 \%$ of all cases. ${ }^{5-7}$ Interestingly, the number of publications addressing this issue is relatively low-given its relevance. There were more attempts to optimize respiratory-averaged $\mathrm{CTs}^{8}$ or to acquire several CT scans and then pick the optimal matching one, but due to the increased exposure to ionizing radiation this appears to be suboptimal. ${ }^{9}$

We think it is fair to assume that the technique of choice today is the manual alignment between PET and CT. Careful quality control and strict rules on how to perform the manual registration are required for reproducible and reliable results but manual interactions are always prone to failure. The study by Slomka et al addresses this critical issue with a software-based approach in 82-Rb cardiac PET/CT using invasive catheterization as gold standard. ${ }^{10}$ Interestingly, they conclude that the optimal strategy is to perform a rigid alignment only in those cases which were identified as misaligned by means of visual assessment. This raises an interesting point: the technologist must be able to rapidly detect such a misalignment. As PET scanner user interfaces are focused on non-cardiac scans, such a suitable visualization is not necessarily available-although it would be very valuable. Surprisingly, the rate of unsatisfactory alignments of the data acquired "as is" is rather high with around 51\% (stress) and 55\% (rest). This outlines again the necessity of strict quality control and proper registration. An additional interesting finding is the fact that the rigid alignment was superior to the elastic approach using a semi-commercial tool box. This hints in the direction that advanced algorithms work better in a somewhat controlled research settings but the clinical robustness might require a reduced level of complexity. Slomka et al also report that many mismatches were observed although proper breathing commands were given. ${ }^{10} \mathrm{We}$ completely agree with the fact that end-expiration is the proper position but we found that training of the patient or even the absence of any breathing command is the good preparation for such a scan as quite a number of patients interpret such a command as the last chance to inhale and then stop breathing.

In addition to an improvement in diagnostic accuracy in static imaging, accurate and ideally automatic attenuation correction is relevant in dynamic imaging as well. ${ }^{11}$ Absolute quantification e.g., of absolute myocardial blood flow showed advantages in diagnosis, 


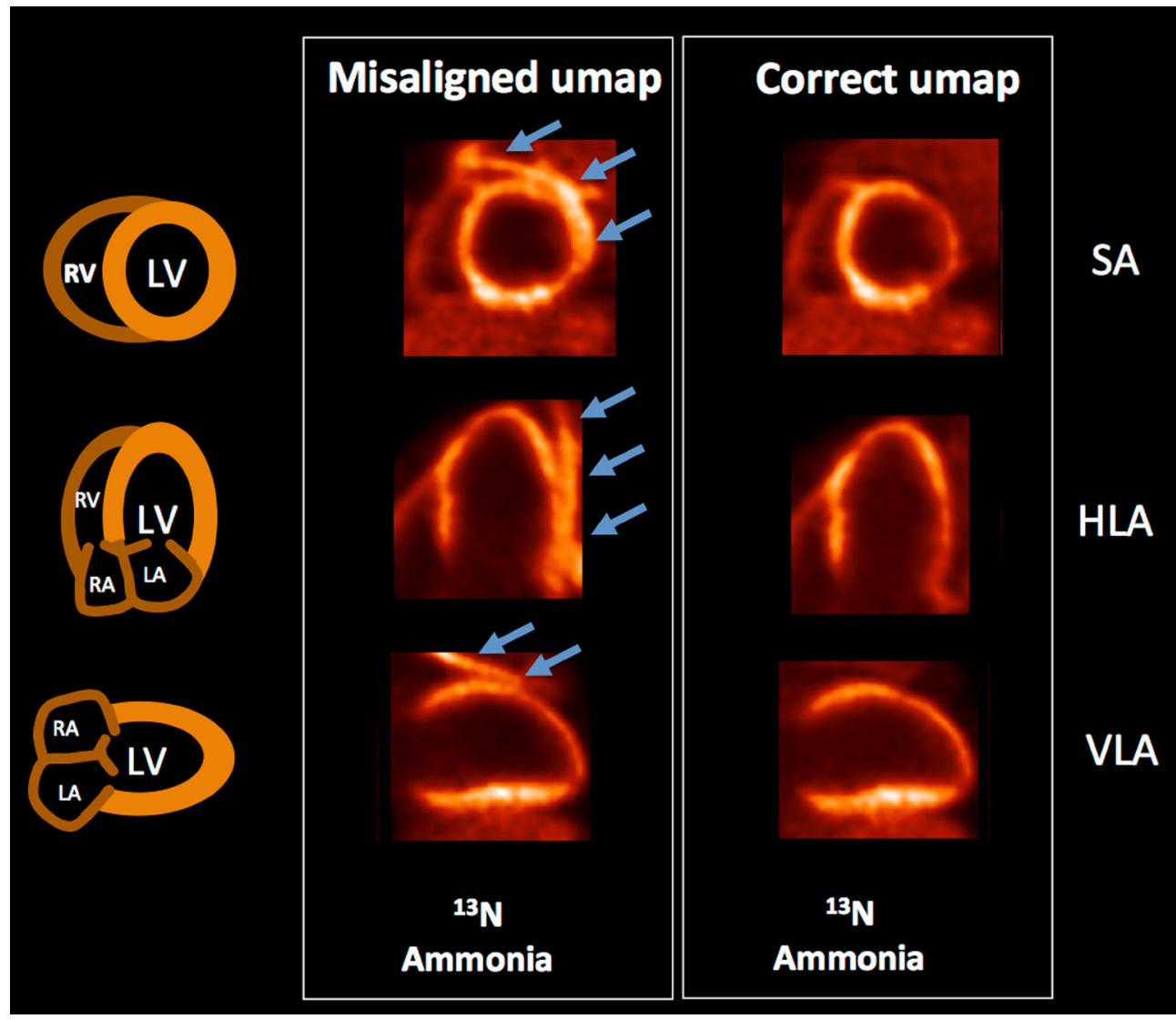

Figure 2. Example of a cardiac ${ }^{13} \mathrm{NH}_{3}$ PET/MRI scan with a misaligned MR-derived $\mu$ map and the scan after correcting the $\mu$ map for misalignment $(6 \mathrm{~mm})$. In this case, a significant lung uptake of ${ }^{13} \mathrm{NH}_{3}$ was present and was projected onto the chest wall-lung interface fully obscuring the reduced perfusion in the lateral wall of this infarct patient.

prediction, and monitoring of cardiovascular diseases. ${ }^{12}$ Thus, a reduction of artifacts is an important element. In dynamic imaging, patient motion might occur anytime and the time after the end of the pharmaceutical stress test appears to be especially vulnerable to it. In such a setting where motion correction has to be performed in 10 or 20 frames, manual procedures are tedious and might become unreliable. Thus, automated processing becomes especially important in such applications.

A similar argument is applicable to dual gated PET/ CT scans where both the respiratory and the cardiac motion are measured and compensated. These acquisition modes might show their potential in the accurate delineation of tracer uptake in the coronaries. ${ }^{13}$ However, in such a setting, an image-based registration will most likely be replaced by elastic registration, ideally incorporated in the image reconstruction to make optimal use of all emission events acquired. ${ }^{14,15}$ An alternative to rigid or elastic registration of the CT would be the modification of the CT to achieve consistent results. ${ }^{5,16}$
It is also worthwhile pointing out that the general problem is not limited to PET/CT but affects also cardiac PET/MR. In this latest addition to nuclear cardiology's armamentarium, an MR scan is converted to a $\mu$ map for attenuation correction. ${ }^{17} \mathrm{~A}$ scan repetition is simple as it is fast and is not associated with ionizing radiation, but the need to make this decision after identifying misalignment is still there. However, we and others found that the $\mu$ map scan in cardiac PET/MR is typically well aligned. ${ }^{18,19}$ The reason is threefold: MRI is a motion-sensitive technique and an elastic belt is the standard equipment. In addition, cardiac MRI is typically acquired in end-expiration so the patient is already used to keep this position. Finally, as the respiratory motion is permanently displayed on the scanners console, it is easy to confirm the patient's respiratory status. Still, as Figure 2 shows, misalignment is possible and results in the same and potentially severe artifacts as in PET/CT.

To conclude, accurate attenuation correction is vital for static, dynamic, and acquisition schemes which are 
even more complex. Unfortunately, most of the advanced correction schemes are still in the domain of research institutions but approaches which appear capable for routine use are starting to appear. ${ }^{20}$ As the number of competing imaging modalities is increasing, substantial efforts such as the work by Slomka et al are needed to minimize the number of artifacts in cardiac PET in order to maintain its full potential.

\section{References}

1. Slomka PJ, Patton JA, Berman DS, Germano G. Advances in technical aspects of myocardial perfusion SPECT imaging. J Nucl Cardiol 2009;16:255-76.

2. Kinahan PE, Townsend DW, Beyer T, Sashin D. Attenuation correction for a combined 3D PET/CT scanner. Med Phys 1998;25:2046-53.

3. Beyer T, Townsend DW, Brun T, Kinahan PE, Charron M, Roddy $\mathrm{R}$, et al. A combined PET/CT scanner for clinical oncology. J Nucl Med 2000;41:1369-79.

4. Souvatzoglou M, Bengel F, Busch R, Kruschke C, Fernolendt H, Lee D, et al. Attenuation correction in cardiac PET/CT with three different CT protocols: A comparison with conventional PET. Eur J Nucl Med Mol Imaging 2007;34:1991-2000.

5. Martinez-Moller A, Souvatzoglou M, Navab N, Schwaiger M, Nekolla SG. Artifacts from misaligned CT in cardiac perfusion PET/CT studies: Frequency, effects, and potential solutions. J Nucl Med 2007;48:188-93.

6. Gould KL, Pan T, Loghin C, Johnson NP, Guha A, Sdringola S. Frequent diagnostic errors in cardiac PET/CT due to misregistration of CT attenuation and emission PET images: A definitive analysis of causes, consequences, and corrections. J Nucl Med 2007;48:1112-21.

7. Lautamaki R, Brown TL, Merrill J, Bengel FM. CT-based attenuation correction in (82)Rb-myocardial perfusion PET-CT: Incidence of misalignment and effect on regional tracer distribution. Eur J Nucl Med Mol Imaging 2008;35:305-10.

8. Nye JA, Hamill J, Tudorascu D, Carew J, Esteves F, Votaw JR. Comparison of low-pitch and respiratory-averaged CT protocols for attenuation correction of cardiac PET studies. Med Phys 2009;36:1618-23.

9. Gould KL, Pan T, Loghin C, Johnson NP, Sdringola S. Reducing radiation dose in rest-stress cardiac PET/CT by single poststress cine CT for attenuation correction: Quantitative validation. J Nucl Med 2008:49:738-45.
10. Slomka PJ, Diaz-Zamudio M, Dey D, Motwani M, Brodov Y, Choi D, et al. Automatic registration of misaligned CT attenuation correction maps in Rb-82 PET/CT improves detection of angiographically significant coronary artery disease. J Nucl Cardiol 2015. doi:10.1007/s12350-014-0060-9.

11. Pourmoghaddas A, Klein R, deKemp RA, Wells RG. Respiratory phase alignment improves blood-flow quantification in Rb82 PET myocardial perfusion imaging. Med Phys 2013;40:022503.

12. Takx RA, Blomberg BA, Aidi HE, Habets J, de Jong PA, Nagel E, et al. Diagnostic accuracy of stress myocardial perfusion imaging compared to invasive coronary angiography with fractional flow reserve meta-analysis. Circ Cardiovasc Imaging 2015;8:e002666.

13. Delso G, Martinez-Moller A, Bundschuh RA, Nekolla SG, Ziegler SI, Schwaiger M. Preliminary study of the detectability of coronary plaque with PET. Phys Med Biol 2011;56:2145-60.

14. Koivumaki T, Nekolla SG, Furst S, Loher S, Vauhkonen M, Schwaiger M, et al. An integrated bioimpedance-ECG gating technique for respiratory and cardiac motion compensation in cardiac PET. Phys Med Biol 2014;59:6373-85.

15. Lamare F, Le Maitre A, Dawood M, Schafers KP, Fernandez P, Rimoldi OE, et al. Evaluation of respiratory and cardiac motion correction schemes in dual gated PET/CT cardiac imaging. Med Phys 2014;41:072504.

16. Alessio AM, Kinahan PE, Champley KM, Caldwell JH. Attenuation-emission alignment in cardiac PET/CT based on consistency conditions. Med Phys 2010;37:1191-200.

17. Martinez-Moller A, Souvatzoglou M, Delso G, Bundschuh RA, Chefd'hotel C, Ziegler SI, et al. Tissue classification as a potential approach for attenuation correction in whole-body PET/MRI: Evaluation with PET/CT data. J Nucl Med 2009;50:520-6.

18. Nensa F, Poeppel TD, Beiderwellen K, Schelhorn J, Mahabadi AA, Erbel R, et al. Hybrid PET/MR imaging of the heart: Feasibility and initial results. Radiology 2013;268:366-73.

19. Rischpler C, Langwieser N, Souvatzoglou M, Batrice A, Marwick $\mathrm{SV}$, Snajberk J, et al. Simultaneous PET/MR imaging in patients early after acute myocardial infarction: Correlation of $18 \mathrm{~F}-\mathrm{FDG}$ uptake and transmurality of late gadolinium enhancement with regional wall motion after 6 months follow-up. Eur Heart J Cardiovasc Imaging 2015 (in press).

20. Zaidi H, Nkoulou R, Bond S, Baskin A, Schindler T, Ratib O, et al. Computed tomography calcium score scan for attenuation correction of N-13 ammonia cardiac positron emission tomography: Effect of respiratory phase and registration method. Int $\mathrm{J}$ Cardiovasc Imaging 2013;29:1351-60. 